TIPA. Travaux interdisciplinaires sur la parole et le langage

32 | 2016

Conflit en discours et discours en conflit

\title{
Intonation et formulation d'un discours polémique sur la question des droits de l'homme
}

Intonation and formulation of a controversial discourse on the issue of human

rights

Mohamadou Ousmanou

\section{OpenEdition}

Journals

Édition électronique

URL : http://journals.openedition.org/tipa/1702

DOI : $10.4000 /$ tipa. 1702

ISSN : 2264-7082

Éditeur

Laboratoire Parole et Langage

Référence électronique

Mohamadou Ousmanou, « Intonation et formulation d'un discours polémique sur la question des droits de l'homme », TIPA. Travaux interdisciplinaires sur la parole et le langage [En ligne], 32 | 2016, mis en ligne le 03 février 2017, consulté le 20 avril 2019. URL : http://journals.openedition.org/tipa/1702 DOI : 10.4000/tipa.1702

Ce document a été généré automatiquement le 20 avril 2019.

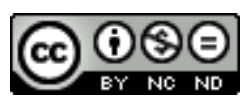

La revue TIPA. Travaux interdisciplinaires sur la parole et le langage est mise à disposition selon les termes de la licence Creative Commons Attribution - Pas d'Utilisation Commerciale - Pas de Modification 4.0 International. 


\title{
Intonation et formulation d'un discours polémique sur la question des droits de l'homme
}

\author{
Intonation and formulation of a controversial discourse on the issue of human \\ rights
}

Mohamadou Ousmanou

\section{Introduction'}

De nombreuses organisations pour la défense des droits de l'homme ont pris l'habitude de publier, de façon régulière, des rapports dans lesquels ils apprécient l'état des droits de l'homme dans différents pays. Ces discours, qui semblent se donner pour fonction de constituer une sorte d'évaluation extérieure de la situation des droits de l'homme, ne suscitent pas que des approbations. Au contraire, ils sont de plus en plus l'objet de critiques et de réfutations de certains hommes politiques qui y voient une manière subtile pour certains États forts d'influencer les autres. Au Cameroun, les médias accordent un intérêt certain à ces débats et donnent aux hommes politiques la possibilité de donner leur avis. Dans l'extrait du débat télévisé dont nous proposons l'analyse ici, le locuteur tend surtout à souligner le fait qu'à travers cette question des droits de l'homme, les pays occidentaux chercheraient à imposer leur vision du monde aux autres. L'objectif du présent article est de montrer comment, en s'appuyant sur les indices intonatifs et discursifs, le locuteur organise son discours de sorte à disqualifier une opinion a priori admise, et travaille, dans le même temps, à s'attirer la sympathie/l'adhésion de ses interlocuteurs. 


\section{Contextualisation et objet de l'étude}

\subsection{Le contexte}

Au cours du mois de mars 2014, le Département d'État des États-Unis publie son rapport sur l'état des droits de l'homme au Cameroun pour l'année 2013². La violence policière, les arrestations et les conditions d'incarcération figurent parmi les principales critiques adressées aux autorités camerounaises. Le contexte est surtout marqué par des problèmes sécuritaires, notamment aux frontières septentrionales. Les journaux traitent de sujets assez divers, mais tendent tous à pointer des questions politiques sérieuses. Dans la liste qui suit, nous reprenons quelques titres à la une des journaux les plus cités lors de la revue de presse de la semaine dans l'émission que nous étudions, Canal Presse:

- Cameroon Tribune : «Parlement. La rentrée. », «Le satisfecit du parlement »

- Le Messager : «Paul Biya maintient des sénateurs cumulards en poste », « Des Camerounais à la tête du chantier naval»

- Le Jour: "Questions autour du poste de vice-président du Cameroun », "Libération de Michel T. Atangana. Paul Biya a cédé aux pressions de la France », « Rapport des États-Unis sur les droits de l'homme au Cameroun »

- L'CFil du Sahel : «Touboro. Des assaillants armés font trois morts »

De toutes ces unes, seuls les titres des derniers journaux sont abordés dans le débat lors de l'émission. Parmi les points discutés dans les débats médiatiques, on peut retenir l'opération de lutte contre la corruption qui a abouti à l'arrestation de certaines personnalités politiques; personnalités qualifiées par certains journaux de prisonniers politiques.

\subsection{Le corpus et la démarche}

Le discours que nous nous proposons d'analyser est issu de Canal Presse, une émission télévisée populaire au Cameroun ${ }^{3}$. Il s'agit d'un débat télévisé qui regroupe des journalistes et des hommes politiques. Dans l'extrait de 2 minutes 16 secondes retranscrit ci-dessous, l'homme politique Saint-Eloi Bidoung, en qualité d'invité, répond à la question qui lui est posée par le présentateur Bouba Ngomna. Nous transcrivons le corpus suivant les conventions de transcription graphique à la perception établies par M.-A. Morel \& L. Danon-Boileau $(1998)^{4}$. Les tracés mélodiques sont obtenus à partir du logiciel PRAAT ${ }^{5}$ qui présente les courbes du fondamental (Fo) en Hertz et de l'intensité (I) en décibels. Sur la figure, la courbe de I est en vert et en haut, tandis que la courbe de F0 est en noir et en bas. Les lignes de marges permettent de démarquer les variations et de situer les plages intonatives ${ }^{6}$.

L'étude recourt à la grammaire de l'intonation pragmatique à laquelle M. Rossi (1999: 57) assigne trois fonctions essentielles : «établir le lien avec le ou les allocutaires (fonction appellative), exprimer un contenu de nature expressive (fonctione expressive) et hiérarchiser l'information du message (fonction représentative).». Les paramètres intonatifs pris en compte sont la fréquence fondamentale (Fo), l'intensité (I), les allongements des syllabes finales et les pauses silencieuses. Le cadre interprétatif proposé par M.-A. Morel \& L. Danon-Boileau (1998) nous semble adéquat pour appréhender, du point de vue énonciatif, les différentes variations qui affectent ces paramètres. Nous 
faisons également appel à la marco-syntaxe de Cl. Blanche-Benveniste (2010) dont le modèle de représentation des structures morphosyntaxiques en paradigmes offre l'avantage de mettre en relief l'agencement et les emboitements de segments discursifs.

\subsection{Transcription du corpus}

Bouba : quand vous parcourez e:::: ce rapport $\{36\}$ y a-t-il une ou deux choses à redire comme le:: $\{35\}$ euh disait euh:: Parfait Siki sur ce rapport

Saint-Eloi : monsieur Bouba Ngomna $\{106\}$ euh::: je ne sais pas quel est le quotient intellectuel de monsieur Alpha Blondy $\{62\}$ mais je présume qu'il doit être très élevé dans la mesure où dans une chanson $\{60\}$ il nous a dit la démocratie des plus forts $\{55\}$ est toujours la meilleure si vous traduisez ça $\{46\}$ en français facile $\{52\}$ ça signifie qu'on vous fait pipi sur les genoux et vous demande de dire qu'il pleut et vous devez dire $\{33\}$ qu'il pleut $\{275\}$ ceux qui s'érigent aujourd'hui $\{63\}$ en:: donneurs de leçons en::: redresseurs de torts $\{37\}$ en::: $\{127\}$ distributeurs de bons et de mauvais points $\{53\}$ ont certainement des problèmes avec les droits de l'homme eux-mêmes $\{214\}$ je voudrais quand même vous dire $\{32\}$ que ${ }^{C^{\prime} E S T}$ à ciel ouvert qu'à Guantanamo \{\} on a vu des hommes violés [toux] on a vu des hommes sodomisés \{38\} on a vu des hommes brûlés vifs au ciagre de Havane \{91\} fût-il roulé sur des cuisses de jeunes filles::: $\{34\}$ vierges $\{47\}$ mais c'n'était pas des bonnes images euh::: nous n'allons pas parler d'Alcatraz nous n'allons pas parler de Miami \{\} nous n'allons pas parler de Noriega $\{57\}$ nous n'allons pas parler de Pablo Escobar $\{65\}$ donc ceux-là ont des problèmes avec les droits avec les droits de l'homme parlant de::: $\{40\}$ euh:: toujours de ces droits \{\} je voudrais même quand vous dire que euh::: des héros africains ont été sauvagement assassinés tout simplement parce qu'ils voulaient exprimer le premier des droits de l'homme $\{135\}$ le ${ }^{\text {DRoIT }}$ à la vie \{\} vous êtes d'accord avec moi que monsieur Lumumba n'est pas mort d'accident de la circulation $\{77\}$ vous êtes d'accord avec moi que monsieur Sankara n'est pas mort du palu $\{75\}$ vous êtes d'accord avec moi que heu Moumié Um Nyobe Ossende Afana ne sont pas morts de méningite \{109\} Sadat Khadafi hier §alors§ vous voyez un peu c ça pour chuter ça me pose un problème je dis que ce rapport moi ne m'émeut pas dans la mesure où il est rédigé par des hommes qui eux-mêmes n'ont aucun respect pour les droits de l'homme

\section{Stratégies énonciatives et variations intonatives}

Dans les échanges oraux télévisés, les locuteurs sont engagés dans un jeu où les points de vue sont le plus souvent connus d'avance ${ }^{7}$. Il s'agit alors pour les intervenants d'anticiper et de se positionner par rapport à une opinion connue. Il semble que ce jeu d'anticipations soit comparable aux opérations coénonciative et colocutive telles que décrites par M.-A. Morel.

Pour analyser les propriétés intonatives des propos de celui qui parle, nous avons retenu deux niveaux de représentation à l'égard de celui auquel le discours est adressé : d'une part le niveau de la coénonciation qui reflète les anticipations que fait le parleur eu égard à la réception de son discours et à la pensée qu'il prête à l'autre (attentes, convergence de points de vue, objections possibles...), et d'autre le niveau de la colocution qui traduit les anticipations qu'il peut faire sur une éventuelle prise de parole de l'écouteur. (M.-A. Morel, 2003 : 294).

Ainsi, le parleur est amené quelquefois à effectuer des ajustements dans la relation coénonciative lorsqu'il envisage un désaccord sur l'objet de discours. Sur le plan intonatif, 
la régulation coénonciative s'opère par des variations de la hauteur mélodique, considérée alors comme une deixis vocale. L'intérêt pour l'étude d'un tel discours concerne surtout les procédés stratégiques mis en place par celui qui parle afin de décrédibiliser cette opinion. Nous nous intéressons ici à trois ressources linguistiques qui entrent en jeu dans l'opération de disqualification : la focalisation intonative, les pauses silencieuses et la réduplication des segments discursifs. Au préalable, l'on examinera l'organisation, au plan discursif, du propos du locuteur.

\subsection{Structures des paragraphes intonatifs : alternance et mise en valeur des constituants}

Le propos de Saint-Eloi s'organise autour de cinq paragraphes intonatifs ${ }^{8}$ que l'on délimite à la fois au plan intonatif - chute conjointe de F0 et I, et absence d'allongement et au plan discursif - succession des constituants préambule + rhème (+postrhème). À l'observation, le locuteur fait succéder alternativement une structure étendue à deux constituants (préambule et rhème) à une structure réduite à un constituant (rhème). Ce choix d'organisation discursive procède d'un jeu stratégique visant à mettre en valeur des fragments discursifs donnés. Nous examinerons deux exemples qui illustrent comment les deux modes d'organisation discursive se complètent et se fondent sur une cohérence globale du discours.

Exemple 1

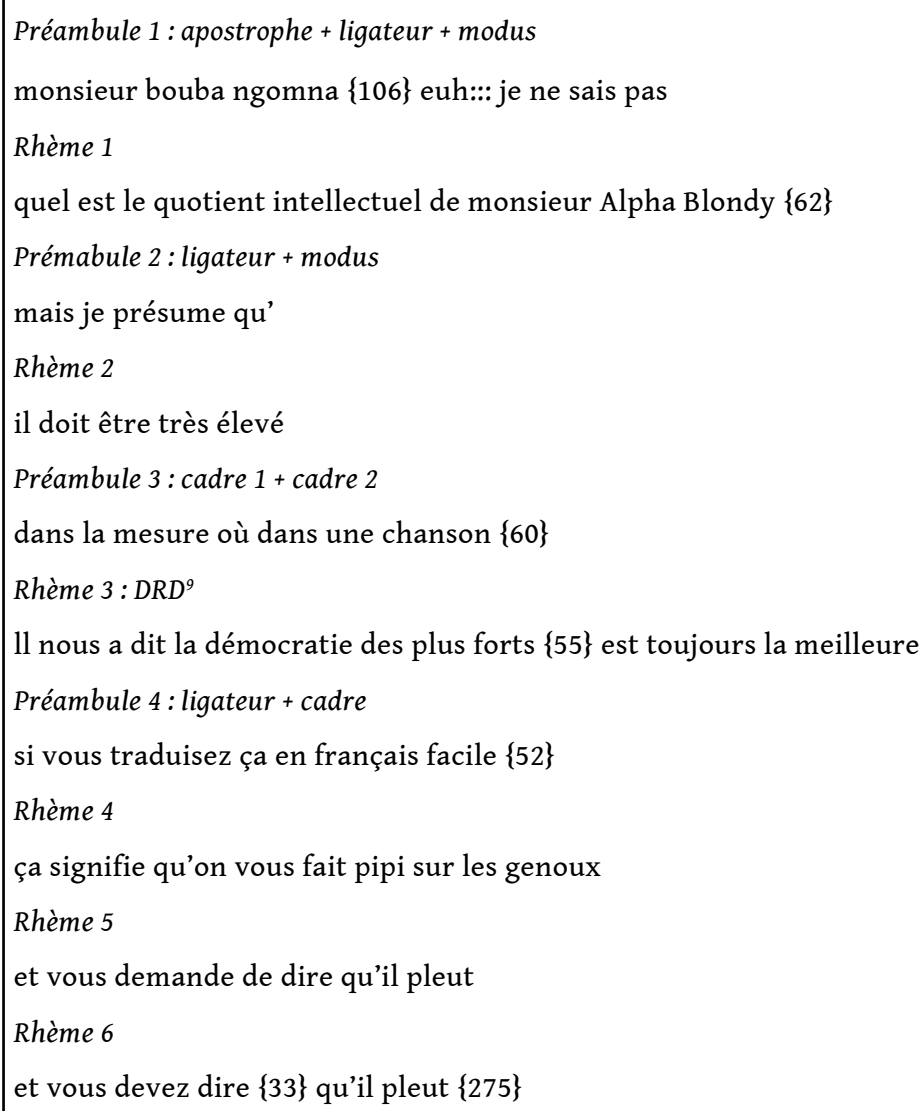


Ce premier paragraphe présente une structure binaire simple : un préambule suivi d'un rhème. Le premier mouvement du propos pose toujours un préambule au préalable. $\mathrm{Ce}$ qui s'explique par le fait que c'est dans ce fragment que le parleur cherche à instaurer un cadre de partage consensuel. Les préambules 1,2 et 3 mobilisent notamment des constituants à valeur modale («je ne sais pas ", « je présume qu' », « dans la mesure où »). Comme on peut le remarquer, le parleur emploie ces marqueurs pour moduler son discours. Même s'il avoue son ignorance à propos du quotient intellectuel d'Alpha Blondy, Saint-Eloi ne s'empêche pas pour autant d'en apprécier la qualité, en recourant à un marqueur du haut degré : «il est très élevé ». Cet aveu qui a l'apparence d'une feinte vise à moduler une prise de position qui contraste radicalement avec celle de ses interlocuteurs. En fait, il ne cite le nom du chanteur qu'à la seule et unique fin de conforter sa thèse de la domination: "la démocratie des plus forts est toujours la meilleure ». Autrement dit, les pays puissants imposeraient leur point de vue aux plus faibles. Ce segment qui correspond au rhème 3 présente la particularité d'être un discours rapporté direct (DRD). Il joue ici un rôle décisif dans la mesure où toute la thèse du locuteur repose sur ce thème de la force et de la victimisation. D'ailleurs, les propriétés intonatives de ce fragment du discours montrent la prise en charge des propos rapportés auxquels il adhère entièrement : remontées de F0 sur le verbe introducteur « dit » et sur l'initiale de l'énoncé du DRD « la démocratie $»^{10}$. Cette condition posée, le locuteur s'attèle à expliciter cette posture par la suite. Il procède par une glose du DRD qui fait succéder les rhèmes 4,5 et 6 .

Cette explication dont le locuteur présente comme une version simple et compréhensible de la citation (" en français facile ») est pourtant loin d'être transparente. Deux énoncés sont mis en correspondance : (a) «la démocratie des plus forts est toujours la meilleure » et (b) «on vous fait pipi sur les genoux et on vous demande de dire qu'il pleut». La relation entre le sens de (a) et celui de (b) est de type analogique. L'équivalence que tente de poser le locuteur ne s'appréhende que de façon implicite. La scène que décrit l'énoncé (a) vise à montrer que quelqu'un, désigné par "on», offense ("fait pipi... sur les genoux») une autre personne, désignée par "vous». En nous intéressant au jeu de pronoms, on peut également voir d'autres correspondances. Le « on » de l'énoncé (b) peut être bien rapproché de « des plus forts » de l'énoncé (a). Il s'agirait alors de ceux qui sont les plus forts qui offensent les plus faibles ("vous») en leur imposant leur vision. Mais une telle interprétation n'est sans doute pas donnée, elle se construit par rapport à la situation énonciative. Aussi l'option de l'explicitation par l'analogie implicite ne paraîtelle pas facilement appréhendable comme l'avance le locuteur ${ }^{11}$. Mais l'enjeu à ce stade, à ses yeux, c'est de poser comme préalable cette idée de la domination. Il procédera, dans la suite de son propos, à un changement qui va réorganiser son discours. 


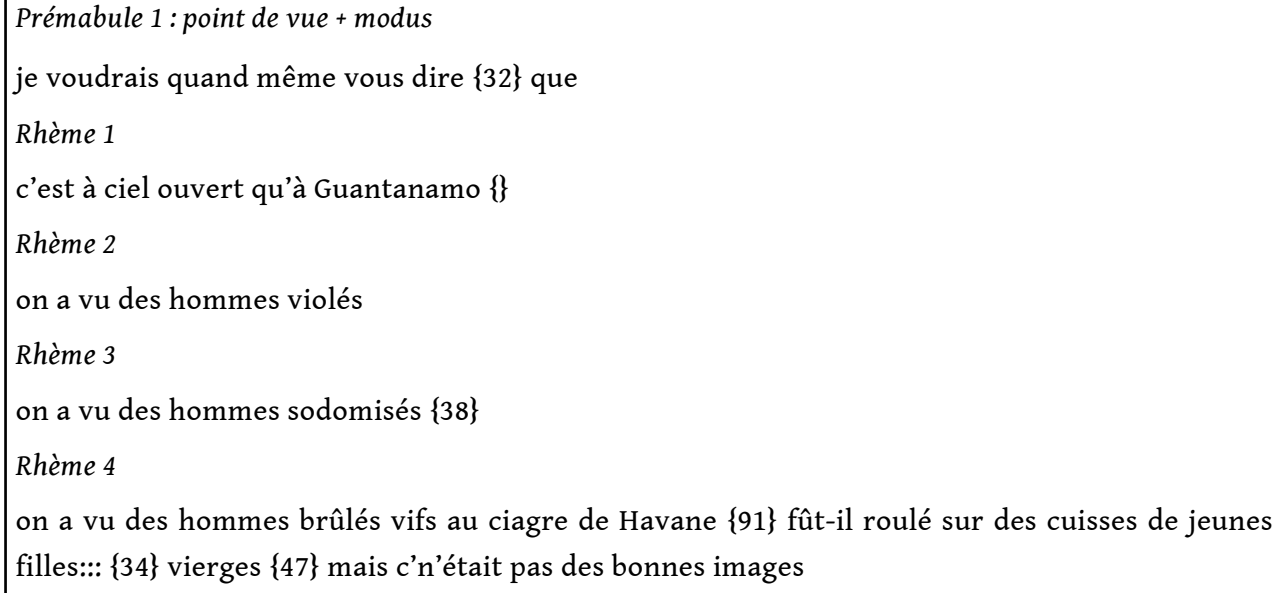

Le passage d'une structure qui alterne préambule et rhème à une structure où se relayent plusieurs rhèmes après un préambule offre au parleur la possibilité d'enchaîner des énoncés qui valident la prise de position énoncée préalablement. L'ensemble précédent prenant alors valeur de préambule, le parleur cherche surtout à faire entériner les accusations qu'il profère. La volonté d'influer sur le jugement de l'autre se confirme au plan intonatif par le rehaussement de Fo à l'initiale de chacun des rhèmes qui se succèdent.

Face à des interlocuteurs à qui il prête un positionnement différent du sien par rapport à l'objet du débat, le parleur est conduit à construire un discours à caractère contestataire afin de déconstruire en quelque sorte ce qui apparaît comme une opinion admise. Un positionnement contraire à ladite opinion passe inéluctablement pour un discours polémique dans la mesure où le point de vue défendu par le parleur n'est pas partagé par tous ses interlocuteurs. P. Charaudeau parle de la position polémique selon laquelle «le sujet argumentant peut choisir des arguments qui vont à l'encontre des valeurs défendues par celui-ci " (1998 : paragr.70). Dans notre contexte, l'enjeu est double pour le parleur. Non seulement il est amené à se représenter les jugements de ses interlocuteurs, qui sont loin de lui être favorables, il doit également se positionner par rapport à une instance du discours, les États-Unis en tant que producteurs du rapport objet du débat, lesquels bénéficient, dans les représentations et les discours qu'en font certains médias, d'une image favorable: pays de liberté, riche et développé, puissance économique et militaire. Mesurant la singularité d'une telle situation, le parleur met en place un discours qui tend à défaire cette représentation positive et en modifier la perception de ceux à qui il s'adresse. Cette opération se ne limite guère à la structuration discursive, elle est aussi repérable au plan prosodique.

\subsection{La focalisation intonative comme procédé de disqualification}

En tant qu'opération énonciative majeure, la focalisation est sollicitée de façon régulière par les locuteurs pour porter un fait à l'attention de l'autre. C'est un procédé qui permet "notamment de rendre saillantes certaines parties du discours (syntagmes, mots, syllabes)» (A. 
Lacheret, 2003 : 1). Dans les échanges oraux, la focalisation est constamment associée à une opération de disqualification.

La focalisation est de façon constante destinée à disqualifier une interprétation erronée que l'on prête à l'autre sur la qualité de l'argument (ou du circonstant) susceptible de valider le prédicat. De ce fait, elle se charge souvent d'une valeur polémique forte. (M.-A. Morel \& L. Danon-Boileau, 1998 : 65).

La disqualification est un phénomène très récurrent dans les débats médiatiques. Par disqualification, nous entendons ici ce procédé énonciatif et stratégique qui consiste à dénier toute crédibilité à une opinion ou à un sujet parlant avec lequel on interagit. Nous avons repéré huit segments de discours, de statut discursif variable, mis en focus dans notre corpus. Leurs propriétés intonatives montrent toujours une démarcation assez nette entre le segment focus et le segment postfocus. Le tableau ci-après montre ces régularités ${ }^{12}$.

Tableau 1 : propriétés intonatives des focus et postfocus

\begin{tabular}{|l|l|l|l|}
\hline Focus & Propriétés intonatives & Postfocus & Propriétés intonatives \\
\hline ceux qui s'érigent & $\begin{array}{l}\text { F0+ (284Hz), I+ (82dB), } \\
\text { précédé d'une pause }(245 \mathrm{cs})\end{array}$ & $\begin{array}{l}\text { en::: donneurs } \\
\text { de leçons }\end{array}$ & $\begin{array}{l}\text { F0+ (287Hz), I+ (79dB), suivi } \\
\text { d'une pause (56cs) }\end{array}$ \\
\hline c'est & F0+ (416Hz), I+ (82dB) & à ciel ouvert & $\begin{array}{l}\text { F0- (75Hz), I- (58dB), suivi } \\
\text { d'une pause (49cs) }\end{array}$ \\
\hline Guantanamo & F0+ (283Hz), I+ (88dB) & on a vu & F0- (75Hz), I- (50dB) \\
\hline $\begin{array}{l}\text { des hommes vio } \\
\text { lés }\end{array}$ & F0+ (276Hz), I+ (82dB) & on a vu & F0- (75Hz), I- (50dB) \\
\hline Sodomisés & $\begin{array}{l}\text { F0+ (288Hz), I+ (83dB), suivi } \\
\text { d'une pause (32cs) }\end{array}$ & $\begin{array}{l}\text { on a vu des } \\
\text { hommes }\end{array}$ & F0- (75Hz), I- (50dB) \\
\hline Brûlés vifs & $\begin{array}{l}\text { F0+ (310Hz), I+ (82dB), suivi } \\
\text { d'une pause (77cs) }\end{array}$ & $\begin{array}{l}\text { au cigare de } \\
\text { Havane }\end{array}$ & $\begin{array}{l}\text { F0- (245Hz), I= (50dB), suivi } \\
\text { d'une pause (85cs) }\end{array}$ \\
\hline des héros & $\begin{array}{l}\text { F0+ (342Hz), I+ (82dB), suivi } \\
\text { d'une pause (46cs) }\end{array}$ & Africains & F0- (169Hz), I- (50dB) \\
\hline Tout simplement & F0+ (345Hz), I+ (82dB) & $\begin{array}{l}\text { parce } \\
\text { voulaient }\end{array}$ & F0- (243Hz), I- (62dB) \\
\hline
\end{tabular}

Les segments mis en saillance font pour la plupart partie du rhème. Le focus est marqué à la fois par une montée de F0 et de I. Dans deux cas, il est suivi d'une pause importante (plus de $20 \mathrm{cs}$ ). Le présentatif c'est qui introduit le rhème "à ciel ouvert " porte le pic intonatif à plus de $400 \mathrm{~Hz}$. La structure rhématique est du type [c'est $X$ que]. Le rôle de l'intensité est ici important dans la mise en relief de l'élément $\mathrm{X}^{13}$. Ainsi, il se crée une véritable rupture avec le groupe suivant qui retombe à $75 \mathrm{~Hz}$ pour $\mathrm{F} 0$ et $58 \mathrm{~dB}$ pour $\mathrm{I}$. De manière régulière, on observe que les postfocus sont dotés d'une mélodie et d'une intensité plus faible. 


\section{Tracé 1}

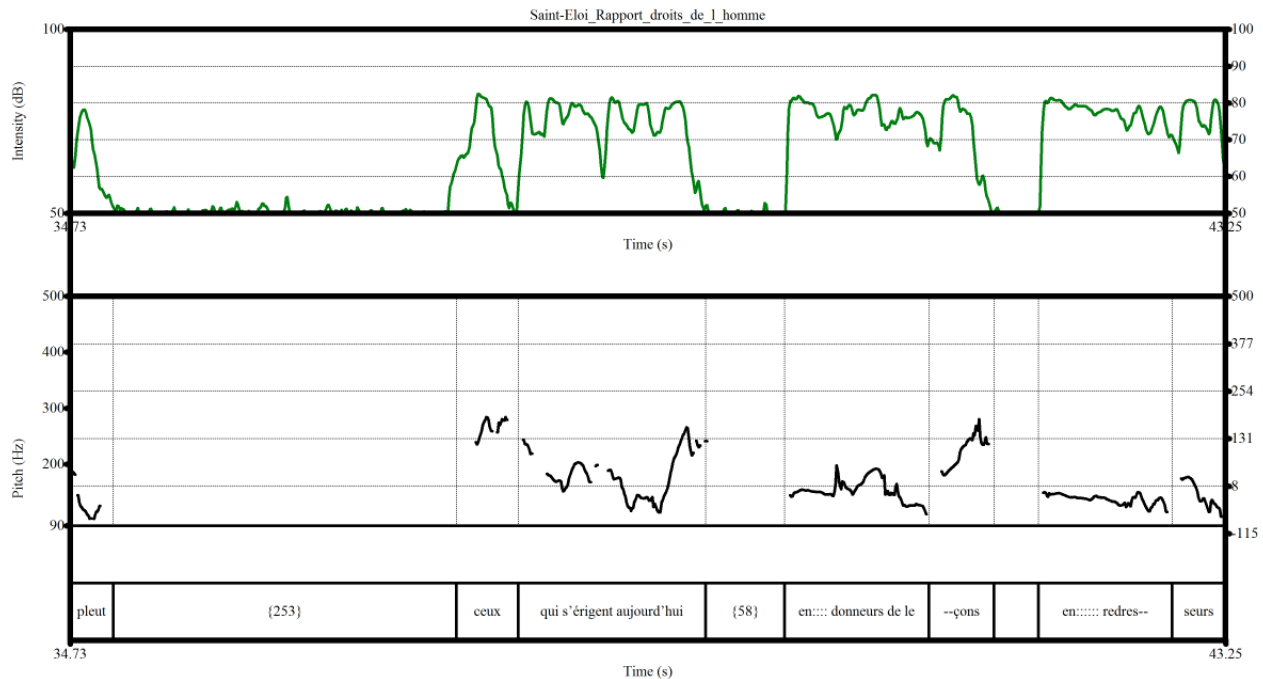

Sur le tracé 1 , on remarque la rupture de la ligne mélodique qui, de part et d'autre de la frontière de la pause, passe de la plage basse à la plage haute. La courbe de F0 connaît alors des modulations allant dans le sens haut-bas-haut (HBH). Puis, on note le retour à un niveau neutre, celui précédant le rehaussement sur " ceux », qui est encore rompu sur la dernière syllabe de « leçons ».

\section{Tracé 2}

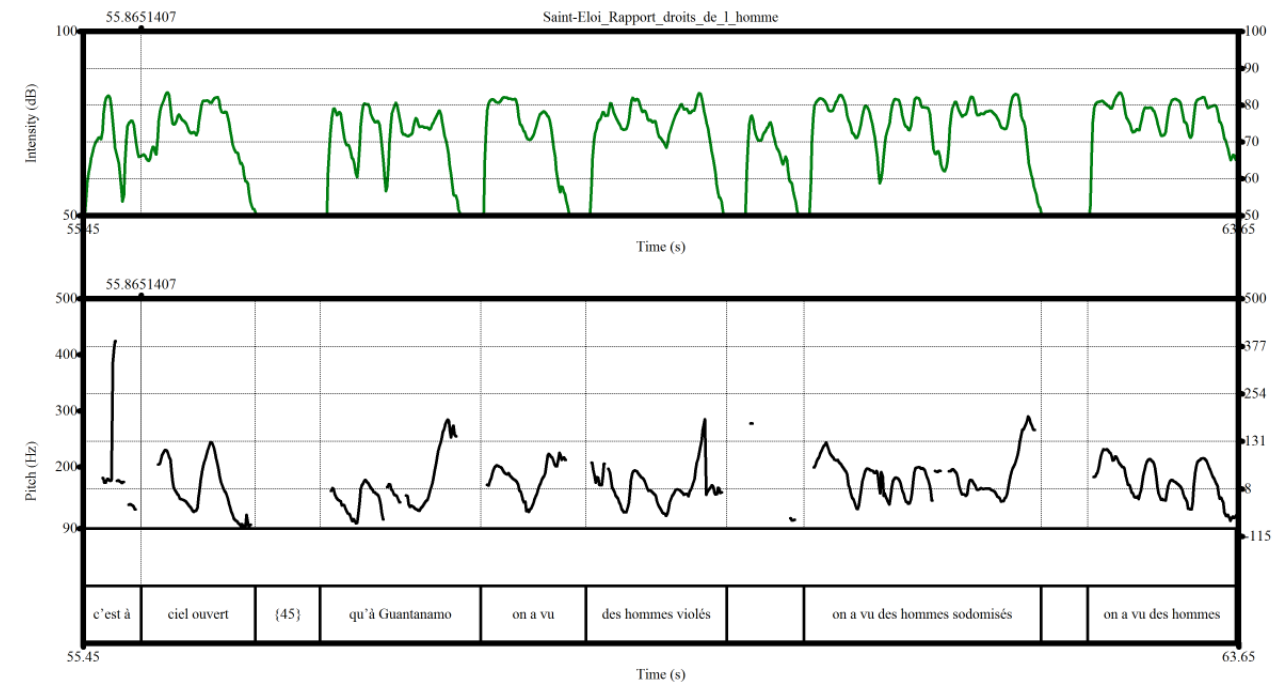

Le tracé 2 présente des modulations plus marquées. Le pic sur "c'est» est le plus nettement visible d'autant plus que le niveau de la courbe de F0 se démarque de tout le reste du segment. Comme sur le fragment précédent, les modulations ont une structure régulière avec une orientation soit en Haut-Bas, ou Bas-Haut ou encore Haut-Bas-Haut. Les rehaussements concernent toujours la dernière syllabe des segments (« GuantanaMO ", « vioLÉS », « sodomiSÉS », « VIFS »). En outre, on remarque l'absence de pause-silence entre les groupes.

En réalité, le discours de Saint-Eloi présente les caractéristiques d'un réquisitoire: énumération des fautes, amplification des faits, dénonciation. La mise en accusation elle- 
même repose sur une liste de faits, connus des interlocuteurs ou supposés comme tels, donc irrécusables. Ce sont les segments discursifs qui supportent ces accusations que le parleur choisit de démarquer à la fois syntaxiquement et intonativement. On note également, à partir de ce site syntaxique précis et contrairement au début de son propos, une accélération du débit chez le parleur. L'absence de pause combinée à l'accélération du débit suggèrent ainsi que ces segments de discours ne présentent pas, aux yeux du parleur, un enjeu pour la discussion dans la mesure où il ne s'attarde pas là-dessus. Ce sont des faits qu'il considère comme non contestables. Le maintien de F0 en plage basse, dès le début de l'intervention, le confirme d'ailleurs. Ce niveau se trouve cependant rehaussé dans le second mouvement du propos («vous êtes d'accord avec moi que Moumié...»). Ce qui peut s'expliquer par le fait que les noms cités dans cette seconde partie ont une valeur énonciative particulière, celle du soulignement contrastif.

L'opération concerne précisément une différenciation que l'énonciateur tient à faire entre le premier mouvement de son propos - où il dépeint les États-Unis de manière négative - et le second - où il met en avant ceux qu'ils nomment " les héros africains ». L'appréciation péjorative des premiers est mise en contraste avec l'image de victime des seconds. Le soulignement se marque, au plan intonatif, par une remontée de F0 sur la syllabe du segment. La mise en saillance des segments est le plus souvent accompagnée des pauses-silences qui permettent de démarquer ainsi certains constituants.

\subsection{La pause silencieuse : durée, place et fonction dans la construction du sens}

S'il est une chose aisément remarquable dans ce discours, ce sont les pauses silencieuses. Elles frappent à la fois par leur nombre (35) et par leur durée (253 cs pour la plus longue, 31 pour la plus petite). Ne sont considérées ici que les pauses de plus de $20 \mathrm{cs}$, seuil qui délimite la pause dite de respiration dont la fonction est essentiellement biologique. Maria Candéa définit la pause silencieuse comme "une interruption signifiative de toute émission sonore à l'intérieur d'une prise de parole ${ }^{14} »(2000: 21)$. La place et la durée des pauses participent de l'organisation locale du discours. Dans leur distinction des pausessilences en fonction de leur durée, M.-A. Morel \& L. Danon-Boileau (1998 : 14) considèrent que les pauses d'une durée située entre $40-80 \mathrm{cs}$ ont une valeur discurive conventionnelle 15. La multiplication des pauses permet au parleur de mettre en saillance certains segments de son discours qui «sonnent » plus que les autres. Par le biais de ces sortes de silences pesants, il arrive à porter à l'attention de l'écouteur ce qu'il veut montrer. Examinons le fragment suivant qui présente les différentes catégories de pauses.

\footnotetext{
ça signifie qu'on vous fait pipi sur les genoux et vous demande de dire qu'il pleut et vous devez dire $\{33\}$ qu'il pleut $\{275\}$ ceux qui s'érigent aujourd'hui $\{63\}$ en:: donneurs de leçons en::: redresseurs de torts $\{37\}$ en::: $\{127\}$ distributeurs de bons et de mauvais points $\{53\}$ ont certainement des problèmes avec les droits de l'homme eux-mêmes $\{214\}$ je voudrais quand même vous dire
}

Deux pauses se démarquent de par l'importance de leur durée. Leur rôle énonciatif est d'opérer un changement dans l'orientation de l'argumentation du parleur. La première pause de 275 cs qui apparaît après la séquence « qu'il pleut » instaure une démarcation qui permet d'introduire la séquence qui suit («ceux qui s'érigent»). La pause permet 
ainsi au parleur de marquer qu'il achève une séquence et de montrer comment il l'articule à une autre. La dernière pause de la séquence, d'une durée encore plus grande (214 cs), marque, encore plus clairement, une rupture au plan thématique avec le segment précédent. Le parleur s'apprête alors à introduire un nouvel objet de discours. Les trois pauses suivantes sont d'une durée importante, respectivement $63 \mathrm{cs}, 37 \mathrm{cs}$ et 127 cs. On peut remarquer que les segments qui suivent ces pauses sont affectés d'allongement sur la préposition « en ». L'apparition de ces allongements montre en effet que le parleur est à la recherche d'une formulation particulière ${ }^{16}$.

Dans ce cas précis, le choix de la préposition le contraint à fournir, sur ce site syntaxique, un nom (" donnneurs de leçons », "redresseurs de torts », "distributeurs de bons et de mauvais points »). Comme on peut le voir sur le tracé 1 , la syllabe allongée se situe au niveau 2 de la plage de $\mathrm{Fo}^{17}$ et avec une forte intensité. Ces pauses participent ainsi de la gestion du sens dans la mesure où le parleur prend le temps de construire le sens de ce qu'il dit et de ce qu'il se prépare à dire. L'idée de la dissimulation du malaise dans la formulation se confirme d'autant plus que le parleur tient à reproduire les mêmes structures et bute par conséquent sur la contrainte syntaxique.

\subsection{La réduplication des segments et l'effet de martèlement}

Le parleur exploite à suffisance la réduplication des structures syntaxiques avec des effets clairement perceptibles au plan intonatif. M.-A. Watine conçoit la réduplication comme une "répétition immédiate et iso-fonctionnelle d'un quelconque segment textuel» (2012:151). Dans les échanges oraux, les locuteurs recourent le plus souvent aux réduplications comme stratégies énonciatives ${ }^{18}$. Saint-Eloi recourt non seulement aux modulations de la mélodie, mais aussi et surtout aux pauses silencieuses qui lui permettent de bien marquer, en variant la longueur de la pause, les segments qu'il souhaite porter à l'attention de ses écouteurs. Les segments rédupliqués soit en trois soit en quatre sont toujours séparés par une pause de durée importante de plus de $20 \mathrm{cs}$. La moins longue de ces interruptions dure 37 cs et vient après " en donneurs de leçons »; tandis que la plus longue dure 65 cs et se situe après «nous n'allons pas parler de Pablo Escobar ». La répétition des segments discursifs constitue non seulement un procédé d'amplification ${ }^{19}$, mais elle est aussi, du point de vue énonciatif, une manière d'agir sur la pensée de l'autre. Pour $\mathrm{Cl}$. Blanche-Benveniste, "le tâtonnement lexical est en lui-même une donnée significative, pour le locuteur et pour celui qui l'écoute» (2010: 138). Dans son discours, Saint-Eloi recourt à ce procédé à quatre reprises relativement dans les mêmes conditions intonatives: modulations de Fo, intensité maintenue en hausse. D'un point de vue discursif, les réduplications portent régulièrement sur les segments rhématiques; le rhème étant le constituant dans lequel le parleur marque sa position différenciée par rapport au coénonciateur. La première de ces réduplications, qui se présente sous la forme d'une structure ternaire, porte sur qualification de l'objet de discours.

ceux qui s'érigent aujourd'hui $\{63\}$ en:: donneurs de leçons

en::: redresseurs de torts $\{37\}$

en::: $\{127\}$ distributeurs de bons et de mauvais points $\{53\}$ ont certainement des problèmes avec les droits de l'homme eux-mêmes 
Les segments rédupliqués ont tous la particularité d'attribuer une qualification négative à ceux qui ont produit le rapport. Le locuteur les présente ainsi comme les maîtres du jeu. Que ce soit le donneur de leçons, le redresseur de torts ou le distributeur de points, ceuxlà sont toujours perçus comme supérieurs aux autres. D'emblée, en alignant ces expressions, le parleur prépare déjà ses écouteurs à ce qui va suivre.

Tracé 3

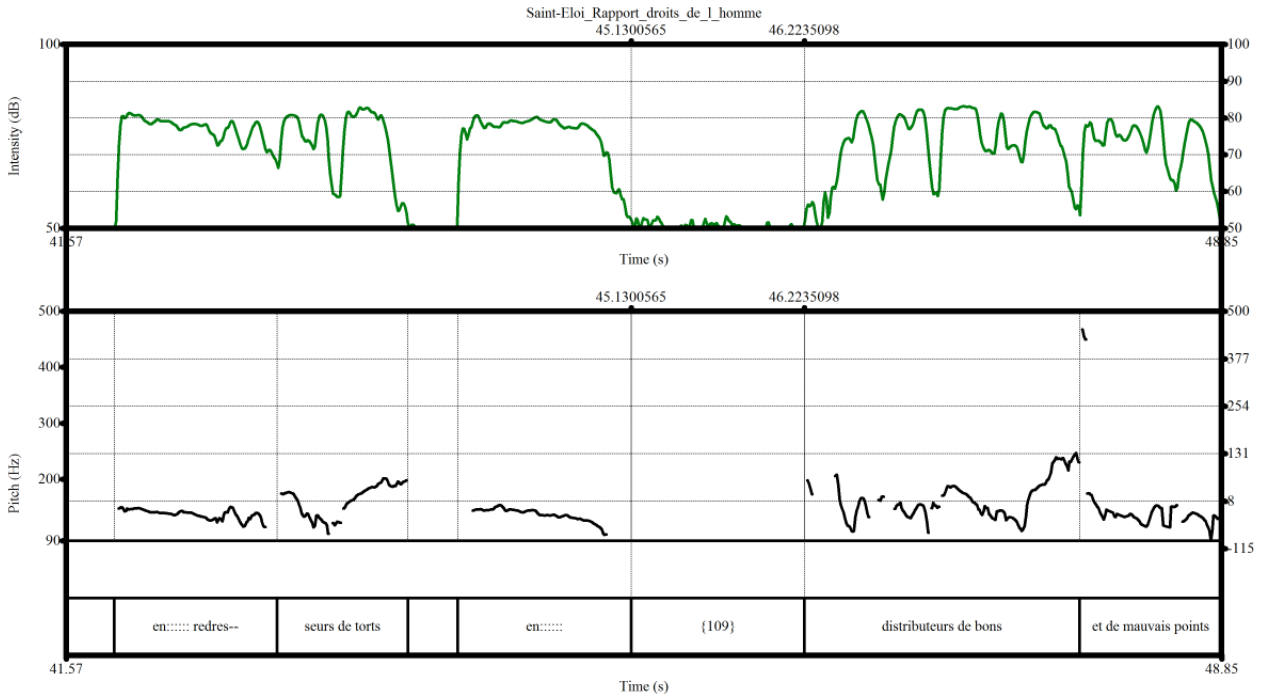

Mais comme nous l'avons souligné plus haut, la réduplication n'apporte pas que des modifications dans la structure discursive. La répétition des segments semblables, pour V. Magri-Bourgues, "modifie le schéma syntaxique et mélodique » (2015: paragr. 10). Le tracé 3 indique quelques modulations de Fo, mais toujours maintenue en plage basse. L'intensité quant à elle reste élevée sur tout le fragment. La conjonction [Fo- ; I+] indique un repli sur soi de la part du parleur, ainsi que sa volonté de conserver la parole.

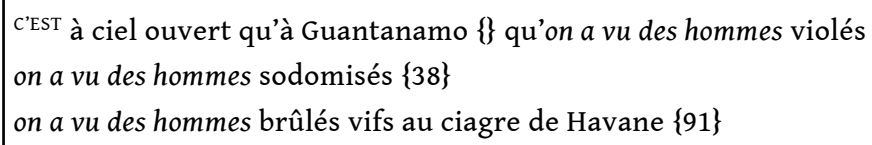

Cette deuxième série de réduplications concerne l'énonciation de contre-exemples visant à montrer que ceux que le parleur désigne par «donneurs de leçons » ne sont pas sans reproches. En fait, le parleur amplifie les faits pour déconstruire l'idée que ceux-là sont supérieurs. De par ces réduplications, le parleur porte à l'attention de ses interlocuteurs des fragments de son discours de manière qu'ils ne soient aucunement matière à remise en question. Il s'ensuit un effet de martèlement et d'accumulation ${ }^{20}$ qui renforce l'opération de décrédibilisation. Saint-Eloi sait pertinemment que l'opinion commune admise veut que les États-Unis soient considérés comme un "modèle » en matière de défense et de promotion des droits de l'homme. Une telle opinion s'impose au jugement de ses interlocuteurs du fait qu'elle relève d'un fond commun. Il s'attèle alors à énumérer les faits qui portent préjudice à ceux-là mêmes qui sont le plus souvent présentés comme le « parangon » en ce qui concerne le respect des droits de l'homme.

La troisième série («nous n'allons pas parler de ») obéit à un fonctionnement similaire. Mais cette fois, ce sont deux noms de lieu (Alcatraz, Miami,) et ceux de deux personnages 
célèbres (Noriega, Pablo Escobar) qui sont évoqués. Par ces simples évocations, le parleur tente d'agir sur la mémoire de ses écouteurs qui sont alors amenés à construire un sens sur ces termes chargés d'histoire : banditisme, criminalité entre autres. La réduplication agit donc sur la mémoire de par les connaissances partagées par les interlocuteurs. Pareillement, le dernier cas de réduplication opère sur l'histoire de l'Afrique.

Tracé 4

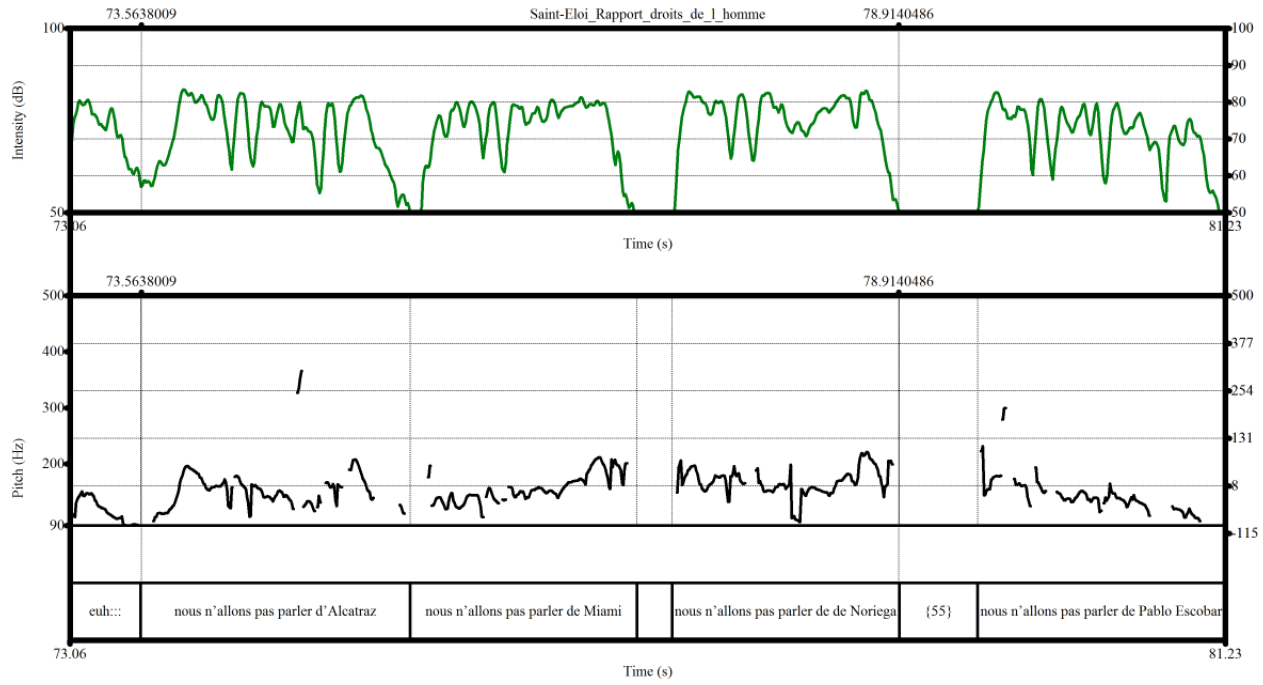

Sur ce tracé, la courbe de F0 est maintenue en plage basse, ne dépassant guère le seuil de $200 \mathrm{~Hz}$. Elle connaît cependant des modulations vers le bas. Ce segment, donné en décrochage par rapport au groupe précédent, présente les caractéristiques intonatives d'une incise ${ }^{21}$ : chute de F0 avec des remontées à la finale du dernier constituant du groupe («Alcatraz», "Miami», «Noriega»), maintien de l'intensité. Ces segments discursifs assument la fonction de cadrage thématique, des informations que le parleur soustrait à toute discussion et qu'il souhaite juste rappeler. En opérant des ruptures de F0 vers le bas, le parleur se met en position d'égocentrage où il se désengage de la coénonciation.

Les séries de réduplications s'enchaînant les unes après les autres, le parleur n'éprouve plus le besoin de prendre en compte les réactions de ses interlocuteurs qu'il a déjà préparés à son discours. Cette sorte d'assurance et de rejet de toute discussion engendre un discours recentré sur son seul point de vue. Cette situation connaît cependant une inflexion dans la suite où le parleur introduit des modulations régulières de F0 sur « Moumié Um Nyobe Ossende Afana », puis sur " d'une méningite ». La conjonction [F0+ ; I-] montre que le parleur est en train de clore une séquence de son discours, mais non pas de son intervention puisque la remontée de F0 a une fonction de recatégorisation des constituants.

parce qu'ils voulaient exprimer le premier des droits de l'homme $\{135\}$ le ${ }^{\text {DROIT }}$ à la vie vous êtes d'accord avec moi que monsieur Lumumba ${ }^{22}$ n'est pas mort d'accident de circulation vous êtes d'accord avec moi que monsieur Sankara n'est pas mort du palu vous êtes d'accord avec moi que heu Moumié Um Nyobe Ossende Afana ne sont pas morts de méningite 
Le dernier exemple semble relever de l'ordre de l'extrapolation. Ceci dans la mesure où les faits énoncés concernent, au plan historique, des colonisateurs européens. Mais il semble que pour le parleur, il ne soit pas nécessaire de distinguer entre une implication directe et une implication indirecte dans ces assassinats. En martelant ainsi ces informations à l'adresse de son interlocuteur, le locuteur cherche à valoriser son seul point de vue tout en disqualifiant celui de l'autre. La réduplication, de par la régularité de la structure discursive, offre cette possibilité d'agir sur la mémoire de l'autre en créant un effet à la fois de captation et d'intensification des faits. On remarquera d'ailleurs que les segments rédupliqués, dans notre contexte, constituent tous des passages où le parleur inclut ses interlocuteurs (" vous êtes d'accord avec moi que », " nous n'allons pas »).

Le jeu de pronoms permet une fois de plus de voir comment le locuteur tente soit d'accorder son point de vue à celui de ses interlocuteurs, soit de le différencier de celui-ci. On distingue des marqueurs d'une position assumée comme «je ne sais pas», «je présume ", des indices tendant à fustiger une instance qu'il ne nomme pas explicitement ("on vous fait pipi», "on vous demande de dire») et des marques d'une volonté d'associer l'autre à son propos («nous n'allons pas parler de », « vous êtes d'accord avec moi que »). L'inclusion, bien évidemment stratégique, participe de cette recherche consensuelle que tente de mettre en place le parleur comme le montrent les modulations de la courbe de F0 vers le haut pour chacun de ses segments (cf. tracé 5 ci-dessous). On peut aussi remarquer que les segments rédupliqués sont essentiellement, du point de vue thématique, des constructions sur des connaissances partagées, ou supposées comme telles.

Tracé 5

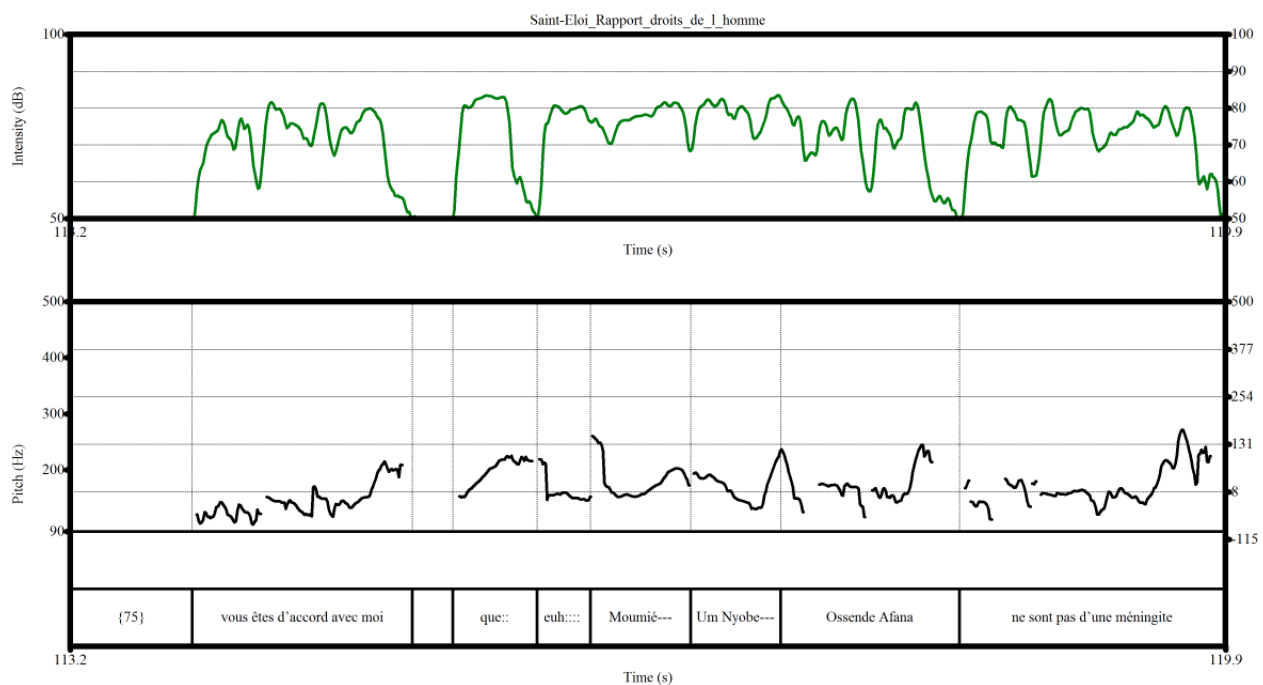

\section{Conclusion}

Cette étude visait à montrer comment, en s'appuyant sur les modulations intonatives et sur les marqueurs de formulation, le locuteur bâtit un discours polémique afin de disqualifier une opinion admise. Son discours, construit à partir de la déconstruction d'un jugement convenu, sonne comme un réquisitoire où il accuse et dénonce. Son propos repose précisément sur l'idée qu'un état, considéré comme fort et en position légitime de 
juger les autres, impose finalement son point de vue. Pour étayer cette thèse, le locuteur opte pour une organisation discursive évoluant d'une structure étendue, lui permettant d'établir un cadre de partage sur la base d'un accord supposé, à une structure réduite, enchaînement des rhèmes mettant en valeur sa seule position. Cette mise en valeur s'opère également par la focalisation intonative qui permet de mettre en saillance des segments précis du discours en leur conférant un statut particulier. De même que la place et la durée des pauses silencieuses, les réduplications soulignent la volonté du parleur d'agir à la fois sur l'attention et la mémoire de ses écouteurs. Il semble que toutes ces ressources linguistiques participent d'une même et unique opération énonciative: la disqualification. Il faut reconnaître que de tels discours se font de plus en plus entendre dans les médias camerounais où journalistes, politiques et politologues animent des débats qui remettent en cause ce qu'ils considèrent être une forme d'impérialisme ${ }^{23}$. Cette étude, qui ne donne qu'un aperçu de la manière dont des hommes et les institutions qu'ils incarnent s'affrontent à travers leurs discours, peut être élargie à d'autres pays africains.

\section{BIBLIOGRAPHIE}

Blanche-Benveniste, Cl. (2010) Approches de la langue parlée en français, Paris, Ophrys.

Candéa, M. \& F. Lefeuvre (2004) Les pics d'intensité dans le récit oral spontané in Lefeuvre, F. \& M. Noailly, Travaux linguistiques du CERLICO 17. Intensité, comparaison, degré 1, p. 27-35.

Candéa, M. (2000) Contribution à l'étude des pauses silencieuses et des phénomènes dits "d'hésitation " en français oral spontané. Étude sur un corpus de récits en classe de français, thèse de doctorat nouveau régime, université Paris III - Sorbonne Nouvelle.

Charaudeau, P. (1997) Les conditions d'une typologie des genres télévisuels d'information, Réseaux vol. 15, No 81, doi : 10.3406/reso.1997.2887, URL : http://www.persee.fr/doc/ reso_0751-7971_1997_num_15_81_2887

Charaudeau, P. (1998) La télévision peut-elle expliquer? in Penser la télévision, Nathan, Paris, URL : http://www.patrick-charaudeau.com/La-television-peut-elle-expliquer.html

Delattre, P. (1966) Les dix intonations de base du français, The French Review, volume 40-1, octobre 1966, p. 1-14.

Lacheret, A. (2003) Focalisation et circonstance, Bulletin de la société de linguistique de Paris, <halshs-00358145>, p. 137-160, URL : https://halshs.archives-ouvertes.fr/halshs-00358145

Magri-Mourgues, V. \& A. Rabatel (2015) Quand la répétition se fait figure, Semen, 38.

Morel, M.-A. \& L. Danon-Boileau (1998) Grammaire de l'intonation. L'exemple du français, Paris, Ophrys.

Morel, M.-A. (2003) Parleur et Écouteur. Formes intonatives couplées dans l'échange oral en français, in Mettouchi, A. \& G., Ferré (coord.) Interfaces prosodiques, Actes du Colloque international de Nantes, mars 2003, p. 27-29.

Rossi, M. (1999) L'intonation, le système du français : description et modélisation, Paris, Ophrys. 
Watine, M.-A. (2012) La réduplication : une interprétation dialogique in Calas, F. et al., Les figures à l'épreuve du discours. Dialogisme et polyphonie, Paris, PUPS.

Watine, M.-A. (2015) Les âges de la réduplication, Semen, 38.

\section{NOTES}

1. Cet article a bénéficié de la lecture et des critiques de Madame Léonie Métangmo-Tatou, directrice du laboratoire LADYRUS. Qu'elle en soit remerciée.

2. Rapport consultable depuis le site du Département d'État des États-Unis, à l'URL: http:// french.yaounde.usembassy.gov/lnsfr_032814.html

3. Emission diffusée le 16 mars 2014. Vidéo disponible depuis https://www.youtube.com/watch? $\mathrm{v}=\mathrm{jN} 0-\mathrm{emnh} 4 \mathrm{UU}$

4. Conventions de transcription : $\{\mathrm{xx}\}$ durée de la pause en centisecondes ; X::: : allongement de la syllabe ; e/euh marqueur d'hésitation ; ${ }^{\circ} \mathrm{XXX}^{\circ}$ : incise (décrochement en plage basse) ; §mm§ : marque d'assentiment de l'interlocuteur; syllabes en petites capitales et en exposant : syllabes dotées d'un pic intonatif ; passages soulignés : superpositions de paroles.

5. PRAAT, version 5.3.82, logiciel de phonétique développé par Paul Boersman et David Weenink de l'Université d'Amsterdam ; URL : www.praat.org.

6. Suivant les principes établis par M.-A. Morel \& L. Danon-Boileau (1998), eux-mêmes inspirés des travaux de P. Delattre (1966), la courbe de F0 est divisée en quatre niveaux : les deux lignes du bas correspondent à la plage basse et les deux lignes du haut représentent la plage haute.

7. P. Charaudeau rappelle à ce titre que les invités dans les débats télévisés sont choisis « en fonction de leur positionnement dans le champ des opinions » (1997:96).

8. Le paragraphe intonatif ou paragraphe oral est l'unité d'analyse de l'oral définie par M.-A. Morel \& L. Danon-Boileau (1998).

9. Discours rapporté direct.

10. M.-A. Morel \& L. Danon-Boileau (1998 : 132) considèrent ces propriétés comme les indices d'une implication totale du parleur dans le discours qu'il rapporte.

11. L'utilisation de l'expression « en français facile " par les journalistes est un phénomène très récurrent dans les débats médiatiques sur lesquels nous travaillons. Cet emploi ne procède pas toujours d'une volonté d'expliciter un propos. Dans de nombreux cas, il se trouve associé à une multiplication de termes relevant du jargon journalistique ou d'un autre domaine technique (sciences politiques, sémiotique).

12. Notation: $F o=$ fondamental en Hertz $(\mathrm{Hz}), \mathrm{I}=$ intensité en décibels $(\mathrm{dB})$, pause en centisecondes (cs). Les symboles + (plus), - (moins) et = (égal) qui suivent F0 et I marquent respectivement une montée, une chute et une stabilité de ces paramètres, affectant les syllabes des segments cités en illustration.

13. Le rôle de l'intensité dans les structures focalisantes a été décrit par M. Candéa \& F. Lefeuvre (2004).

14. Elle précise cependant qu'il existe parfois des pauses silencieuses entre deux prises de parole de deux locuteurs différents, notamment dans le cas d'une conversation (M. Candéa, $2000: 21$ ).

15. Cette classification distingue la pause-respiration $(20 \mathrm{cs})$, la pause d'homogénéisation (40-60 cs), la pause d'annulation et la pause modificatrice du statut discursif du groupe suivant (supérieures à $40 \mathrm{cs}$ ). Pour une étude détaillée des types de pauses et des marques du travail de formulation dans cette perspective, on peut se rapporter à M. Candéa (2000).

16. Sur le rôle de l'allongement syllabique comme marque de travail de formulation, on peut consulter M. Candéa (2000).

TIPA. Travaux interdisciplinaires sur la parole et le langage, 32 | 2016 
17. Le niveau 2 correspond à un repli sur soi ou un face à face selon M.-A. Morel \& L. DanonBoileau (1998: 12).

18. L'interprétation des réduplications comme acte d'énonciation est par exemple présente chez V. Magri (2015).

19. Sur les différentes approches de la réduplication, on peut consulter Marie-Albane Watine (2015).

20. Un tel effet est également étudié par V. Magri-Mourgues (2015).

21. On trouve une description détaillée des caractéristiques intonatives de l'incise à l'oral dans M.-A. Morel \& L. Danon-Boileau (1998), notamment dans le chapitre 5 consacré aux ruptures qui affectent la courbe intonative.

22. Hommes politiques africains: Lumumba (Congolais), Sankara (Burkinabè), Moumié, Um Nyobe et Ossende Afana (Camerounais). Ces hommes sont considérés comme des nationalistes ayant combattu contre le pouvoir colonial en Afrique.

23. Le dernier rapport de ce type a été rendu public par Amnesty International le 14 juillet 2016. De nombreuses réactions, dont celui du ministre de la communication et porte-parole du gouvernement, ont été entendues. On peut lire le discours du ministre depuis http:// www.cameroonvoice.com/news/article-news-25699.html

\section{RÉSUMÉS}

La publication de rapports réguliers sur la question des droits de l'homme dans différents pays du monde a pris une place importante dans les médias au Cameroun. Les journaux proposent des comptes rendus, des débats sont organisés sur les chaînes de télévision. Loin de faire l'unanimité, ces rapports, pour la plupart produits par des institutions occidentales, sont l'objet de nombreuses et vives discussions. Dans cette étude, nous analysons la manière dont un locuteur, grâce aux modulations intonatives et aux marqueurs de formulation, met en place une stratégie énonciative pour disqualifier une opinion communément admise. L'étude, s'inscrivant dans la perspective de l'analyse de l'oral, s'attache à décrire le fonctionnement des indices intonatifs (fondamental, intensité, pause silencieuse) et à en proposer une interprétation énonciative.

In this study, we examine how intonation and formulation markers are used by a speaker to produce a controversial discourse in order to disqualify a commonly accepted point of view on human rights.

The context

The U.S. State Department published a report on human rights in Cameroon in March 2014. This publication occurs in a context especially marked by the security concern, precisely in the northern parts of the country. Newspaper headlines scream "kidnapping of a religious personality in the Far North Region," "creation of the post of vice president." This shows that the context in which the report appears is somewhat tense. Among the points of the report that are discussed through the media, one can retain control operation against corruption which led to the arrests of political figures, arrests deemed not to conform to judicial proceedings.

The corpus and the method

The corpus is made of an excerpt of a two-minute speech by Saint-Eloi Bidoung, a Cameroonian political personality taken from the popular TV show Canal Presse on Canal 2 international channel. This TV show brings together journalists and politicians to discuss issues which appeared in the 
newspaper during the week. In this excerpt, the politician Saint-Eloi Bidoung, from the ruling party, reacts to the question of the presenter, Bouba Ngomna, on how he welcomed the report of the State Department.

Based upon the theory of spoken language analysis (Blanche-Benveniste, 2010, Morel \& DanonBoileau, 1998), the study takes into consideration intonation parameters such as the fundamental frequency (F0) or pitch, the intensity and the pause. These parameters are measured thanks to PRAAT which helps to get pitch and intensity curves. Intonation parameters, according to MaryAnnick Morel \& Laurent Danon-Boileau (1998), play a major role in the functioning of spoken dialogue. This theory defends the hypothesis that, on one hand, the variations of the fundamental how the speaker considers the reception of his discourse by his listener while the variations of intensity point out the way he anticipates turns taking.

Some results

The discourse presents a regular structure with significant pauses separating segments which mostly are reduplicated. The role of $\mathrm{F} 0$ and the pauses is of highlighting these regular segments. When analysed in intonational paragraphs, the discourse of Saint-Eloi Bidoung reveals two type of structuring. First, we notice a binary structure ("préambule + rhème") reduplicated twice. Then another type of discursive organisation follows: "préambule + rhème 1 + rhème 2 + rhème 3. The reduplication of a single type of segment ("rhème") is part of an enunciative strategy based upon a consensus previously established or perceived as such by the speaker with his interlocutors. This consensual minimum acquired, the speaker strives to enhance his only differentiated perspective. This discourse structuring, as well as intonation patterns, is part of an enunciative strategy.

Focalisation

As a major enunciative operation, focalisation is regularly used by the speakers to draw the attention of their interlocutors on certain issues. In lively conversations, focalisation is consistently associated with a disqualification. It is a very recurrent phenomenon in media debates which consist in a strategic process of discrediting a person or a point of view. We identified eight segments set as focus in our corpus. Their intonative characteristics always show a fairly clear demarcation between the segment and the focus postfocus segment.

The pauses

Pauses are easily remarkable in this discourse. They hit both by their number (35) and their length (253 centisecondes for the longest, 31 centisecondes for the shortest). The place and duration of the pause participate in discourse structuring and the construction of the meaning. M.-A. Morel \& L. Danon-Boileau (1998: 14) consider pauses of between 40-80 centisecondes to have a conventional discurive value. By multiplying pauses, the speaker stresses on the segments of his discourse, and therefore manages to focus attention on particular aspects of his indictment of western human rights policy.

Reduplication

The speaker operates large reduplication of structures which are clearly perceptible at intonation level. He resorts to reduplications as enunciative strategy. Saint-Eloi is aware that there is that popular opinion which considers the United States as a model of human rights and freedom land. He then got down enumerating the facts and put the very people who are most often presented as the "paragon." Naming places such as Alcatraz, Miami, Guantanamo and a person such as Pablo Escobar is enough to question that image of perfect model. Reduplication therefore works on the memory, in the domain of the shared knowledge by the interlocutors. It seems that all these language resources, intonation and discourse markers, are involved in one and the same enunciative operation: disqualification. 
INDEX

Mots-clés : intonation, focalisation, pause silencieuse, réduplication, disqualification

Keywords : intonation, focalisation, pause, reduplication, disqualification

\section{AUTEUR}

MOHAMADOU OUSMANOU

Laboratoire LADYRUS - Langues, dynamiques et usages, Université de Ngaoundéré (Cameroun) mohamadusman_06@yahoo.fr 